\title{
3D polarization correlometry of object fields of networks of biological crystals
}

\author{
A.G. Ushenko ${ }^{1}$, A.V. Dubolazov ${ }^{1}$, O.Yu. Litvinenko², V.T. Bachinsky ${ }^{2}$, \\ Lin $\mathrm{Bin}^{3}$, Guo $\mathrm{Bin}^{3}$, Chen Zhebo ${ }^{3}$ \\ ${ }^{1}$ Chernivtsi National University, 2 Kotsiubynskyi Str., Chernivtsi, Ukraine, 58012 \\ ${ }^{2}$ Bukovinian State Medical University, 3 Theatral Sq., Chernivtsi, Ukraine, 58000 \\ ${ }^{3}$ Reasearch Institute of Zhejiang University-Taizhou, 38 Zheda Road, Hangzhou, People's Republic \\ of China, Zhejiang Province, 310027
}

\begin{abstract}
The results of the study of the relationships between 3D divisions of the optical anisotropy parameters of polycrystalline networks of films of biological fluids of various biochemical composition and layer-by-layer phase cross sections of volume distributions of the magnitude and phase parameters of the "two-point" Stokes vector of a microscopic image are presented. In the framework of the statistical approach using scale-selective wavelet analysis, the values and ranges of statistical 1st-4th order changes are defined, which characterize:

- distribution of the values of the modulus and phase of the parameters of the Stokes vector in various phase sections of the object field;

- a set of values of the amplitudes of the wavelet coefficients for various scales of the geometric dimensions of the module maps and the phase of the degree of correlation of the parameters of the Stokes vector (DCS).
\end{abstract}

Keywords: polarization, biological crystals, correlometry, diagnostic.

\section{3D DISTRIBUTION OF THE MODULE AND PHASE OF THE "TWO-POINT" STOKES VECTOR OF THE LIQUOR FILM IMAGE}

The module $\left|S_{4}\right|(\varphi, x, y)$ and phase $\operatorname{ArgS}_{4}(\varphi, x, y)$ of the 4th parameter of the "two-point" Stokes vector, which turned out to be the most sensitive to changes in the optical anisotropy of histological sections of biological tissues, were selected as the main information parameter ${ }^{1-6}$.

Experimental results of 3D Stokes-correlometric mapping with layered digital holographic reproduction of distributions of MDCS $\left(\left|S_{4}\right|(\varphi, x, y)\right.$, fragments (1), (2)) and PDCS ( $\operatorname{Arg} S_{4}(\varphi, x, y)$, fragments (3), (4)) microscopic images of a polycrystalline liquor film according to the method described in ${ }^{7-12}$ are presented in the series of fig. $1(\varphi=0,4 p a \partial)$, fig. 4 ( $\varphi=0,8 \mathrm{rad})$ and fig. $7(\varphi=1,2 \mathrm{rad})$.

In fig. 2, fig. 5, fig. 8 shows the topographic structure (100pix 100pix) of the distributions of the magnitude of the module $\left|S_{4}\right|$ (fragment (1)) and phase $\operatorname{ArgS}_{4}$ (fragment (2)) of the "two-point" 4th parameter of the Stokes vector of the microscopic image of the polycrystalline liquor film obtained for a series of phase cross sections $\varphi=0,4 p a \partial$, $\varphi=0,8 p a \partial$ and $\varphi=1,2$ pad, respectively.

Maps (fragments (1), (4)) and linear sections (fragments (2), (3), (5), (6)) wavelet coefficients of the distribution of the magnitude of the module $\left|S_{4}\right|$ (fragments (1) - (3)) and phase $\operatorname{ArgS}_{4}$ (fragments (3) - (6)) of the "two-point" 4th parameter of the Stokes vector of the microscopic image of the polycrystalline liquor film are shown in fig. 3 (( $\varphi=0,4$ pad ), fig. $6(\varphi=0,8$ pad $)$, fig. $9(\varphi=0,8 p a \partial)$.

Fourteenth International Conference on Correlation Optics, edited by

Oleg V. Angelsky, Proc. of SPIE Vol. 11369, 113691M · (c) 2020 SPIE

CCC code: $0277-786 \mathrm{X} / 20 / \$ 21 \cdot$ doi: $10.1117 / 12.2553942$ 


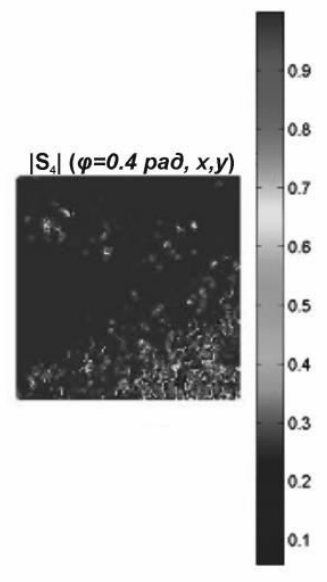

(1)

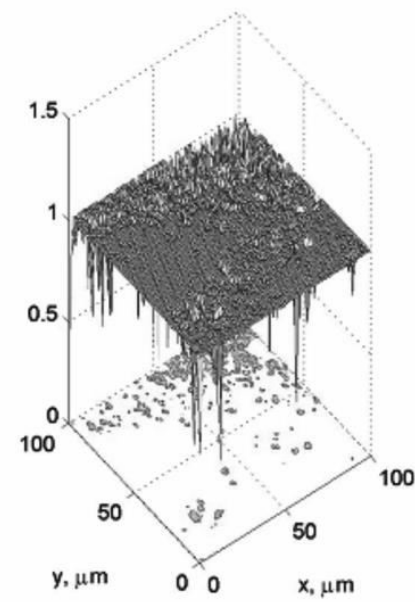

(2)

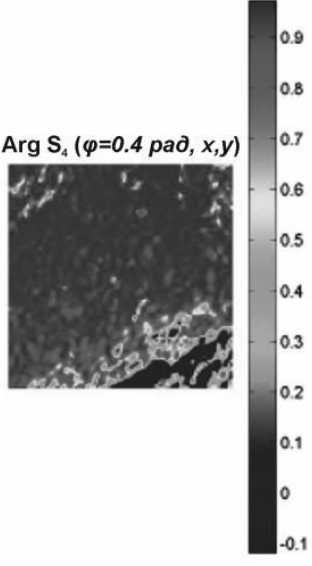

(3)

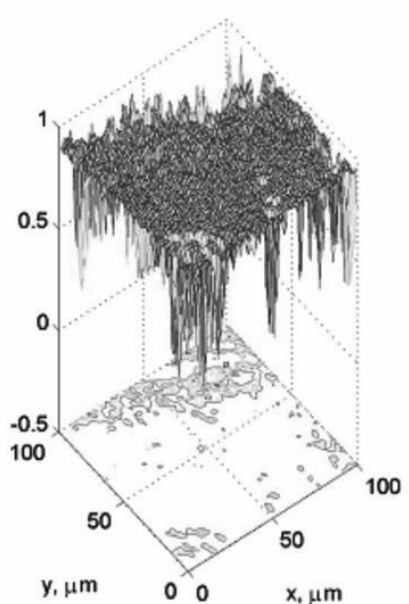

(4)

Fig. 1. Distributions of the magnitude of the module $\left|S_{4}\right|$ (fragments (1), (2)) and phase $\operatorname{ArgS}$ (fragments (3), (4)) of the "two-point" 4th parameter of the Stokes vector of the microscopic image of the polycrystalline liquor film for the phase section $\varphi=0,4$.

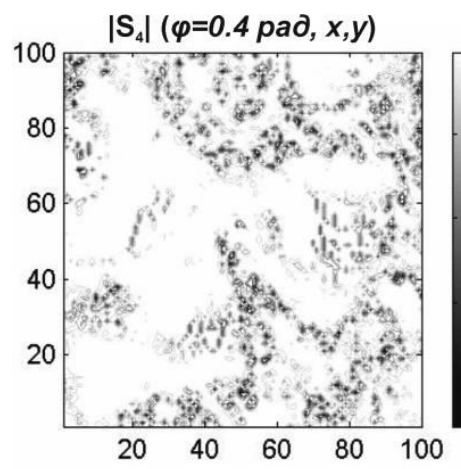

(1)

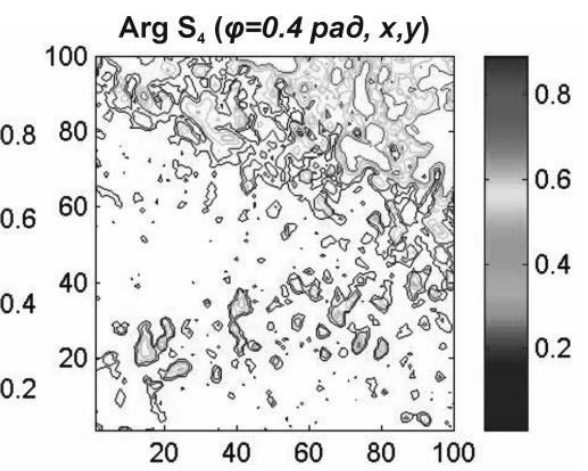

(2)

Fig. 2 Maps (100pix 100pix) of the distribution of the magnitude of the module $\left|S_{4}\right|$ (fragment (1)) and phase $\operatorname{ArgS}_{4}$ (fragment (2)) of the 4th parameter of the Stokes vector of the image of the liquor film for $\varphi=0,4$. 


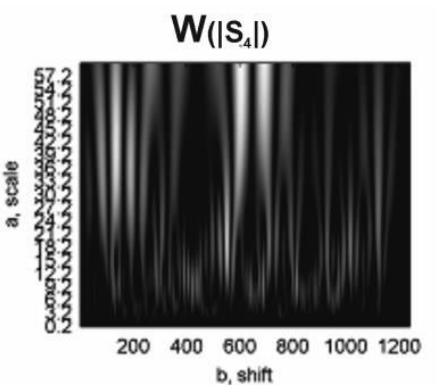

(1)

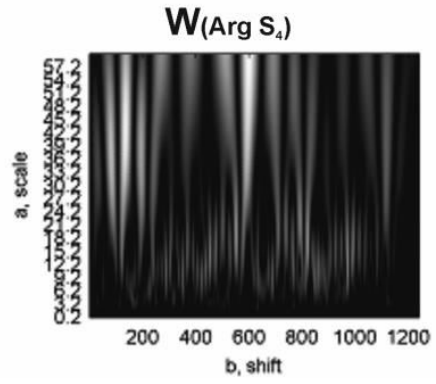

(4)
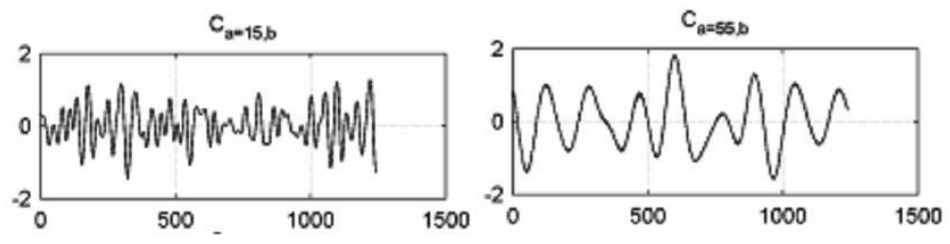

(2)
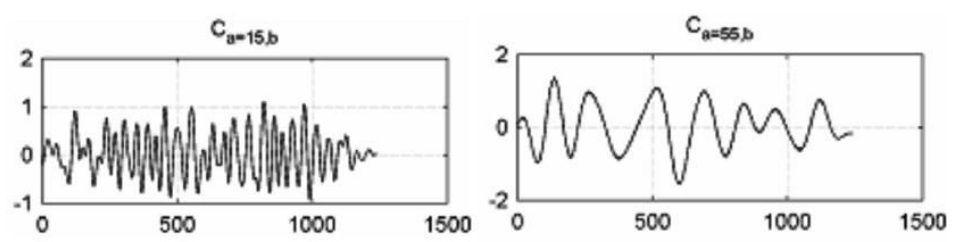

(5)
(3)

(6)

Fig. 3. Maps (fragments (1), (4)) and linear sections (fragments (2), (3), (5), (6)) of the wavelet coefficients of the distributions of the module $\left|S_{4}\right|\left((1)\right.$ - (3)) and phase $\operatorname{ArgS}_{4}$ (fragments (3) - (6)) for $\varphi=0,4$.

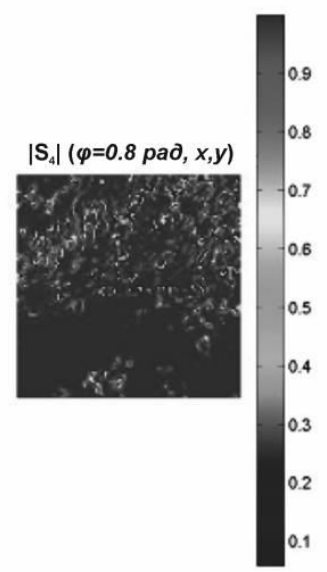

(1)

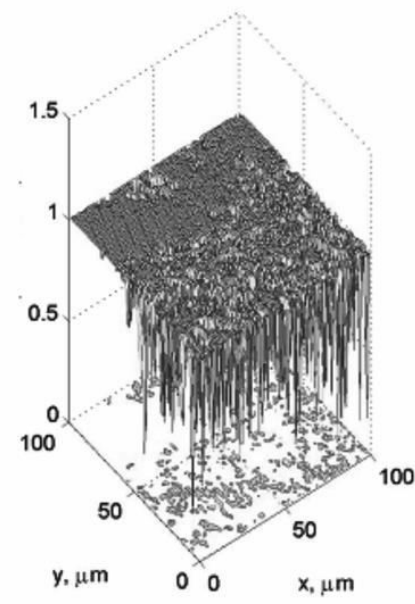

(2)

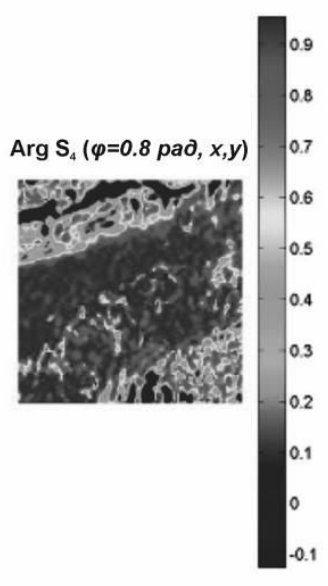

(3)

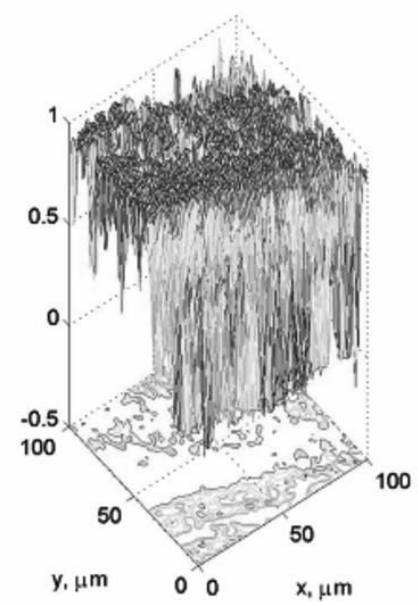

(4)

Fig. 4. Distributions of the magnitude of the module $\left|S_{4}\right|$ (fragments (1), (2)) and phase $\operatorname{Arg} S_{4}$ (fragments (3), (4)) of the "two-point" 4th parameter of the Stokes vector of the microscopic image of the polycrystalline liquor film for the phase section $\varphi=0,8$. 

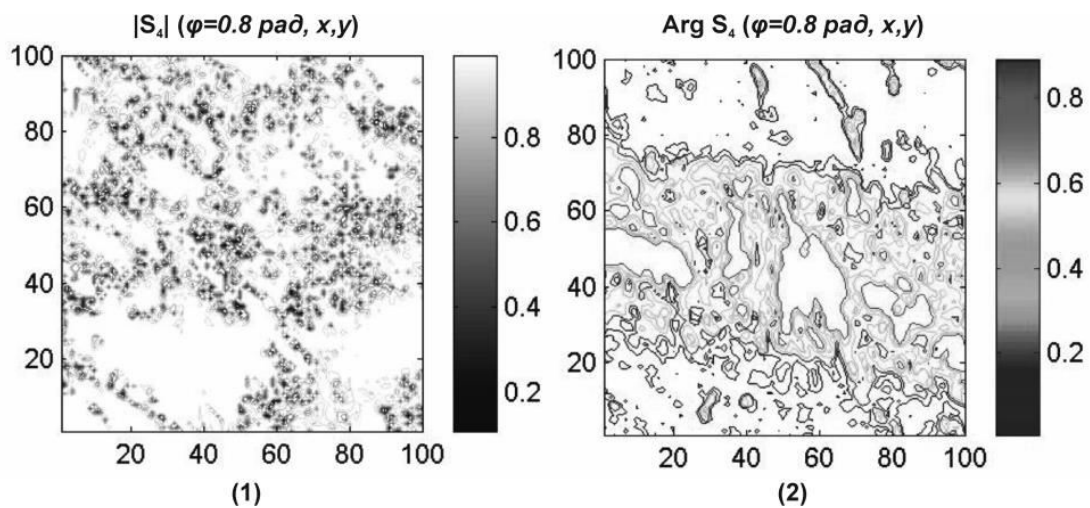

Fig. 5. Maps (100pix 100pix) of the distributions of the magnitude of the module $\left|S_{4}\right|$ (fragment (1)) and phase $A r g S_{4}$ (fragment (2)) for the phase section $\varphi=0,8$.

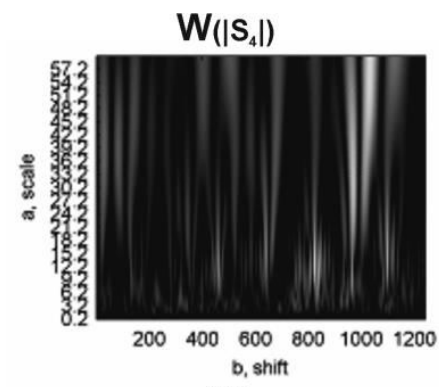

(1)

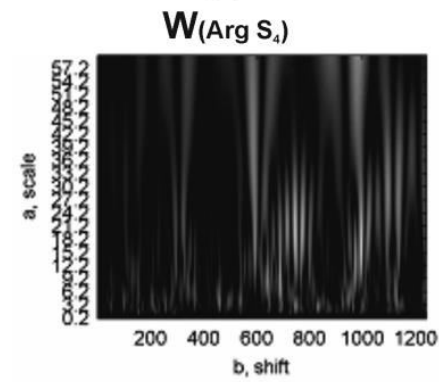

(4)

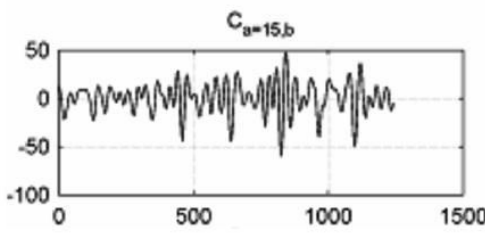

(2)

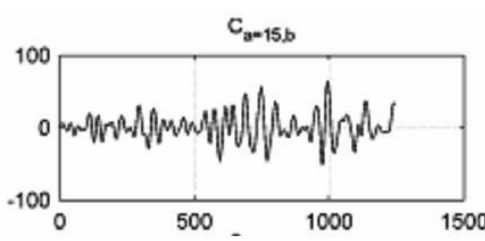

(5)

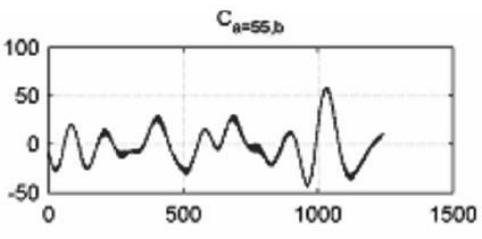

(3)

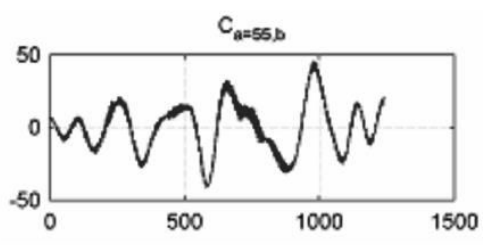

(6)

Fig. 6. Maps (fragments (1), (4)) and linear sections (fragments (2), (3), (5), (6)) of the wavelet coefficients of the distributions of the module $\left|S_{4}\right|\left((1)\right.$ - (3)) and phase $\operatorname{ArgS}_{4}$ (fragments (3) - (6)) for $\varphi=0,8$. 


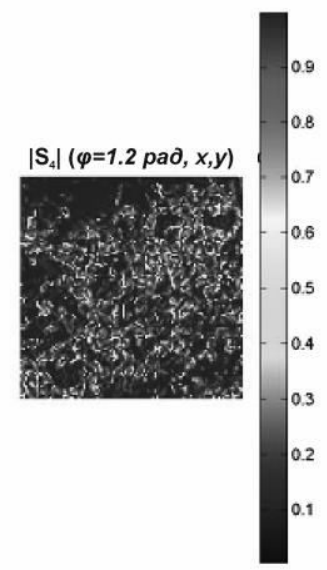

(1)

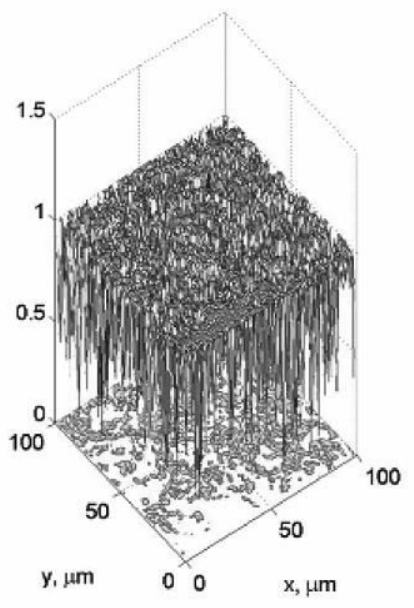

(2)

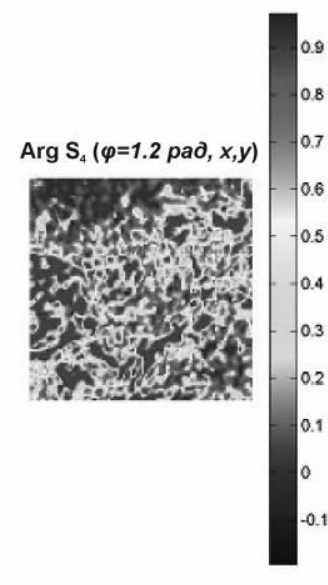

(3)

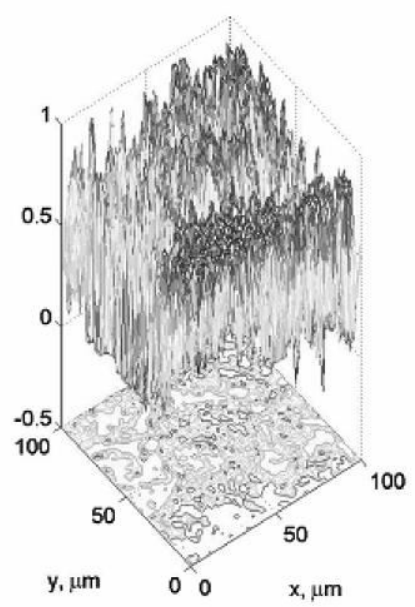

(4)

Fig. 7. Distributions of the magnitude of the module $\left|S_{4}\right|$ (fragments (1), (2)) and phase $\operatorname{Arg} S_{4}$ (fragments (3), (4)) of the "two-point" 4th parameter of the Stokes vector of the microscopic image of the polycrystalline liquor film for the phase section $\varphi=1,2$.

$\left|\mathrm{S}_{4}\right|(\varphi=1.2 \mathrm{pad}, x, y)$

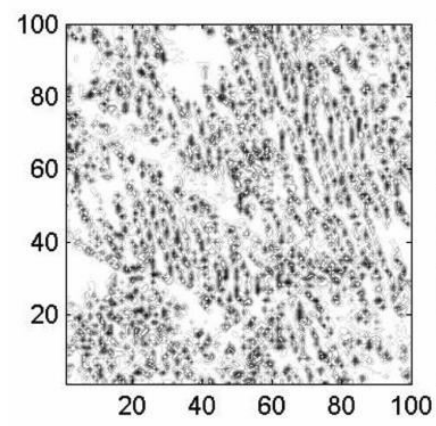

(1)

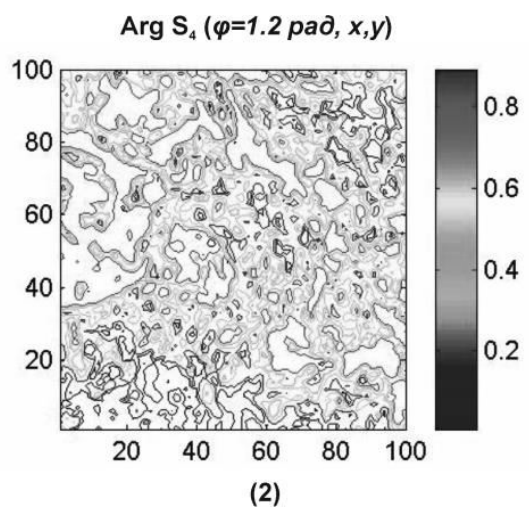

(2)

Fig. 8. Maps (100pix 100pix) of the distributions of the magnitude of the module $\left|S_{4}\right|$ (fragment (1)) and phase $A r g S_{4}$ (fragment (2)) for the phase section $\varphi=1,2$. 


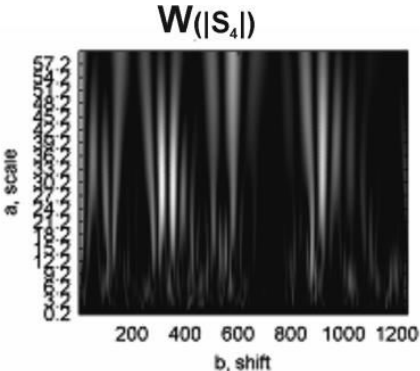

(1)

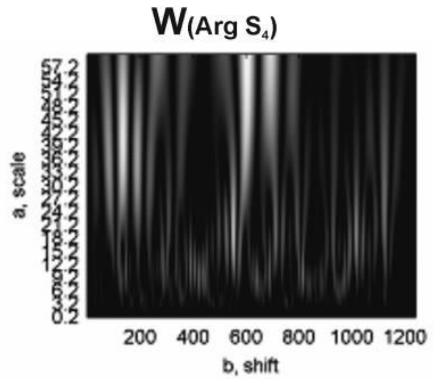

(4)

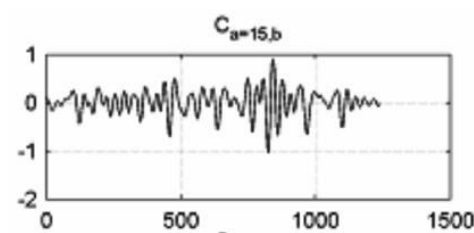

(2)

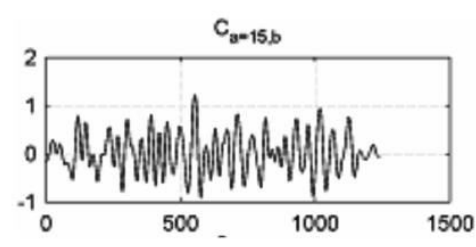

(5)

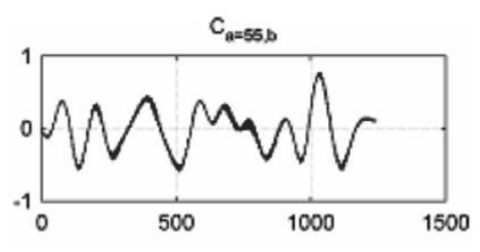

(3)

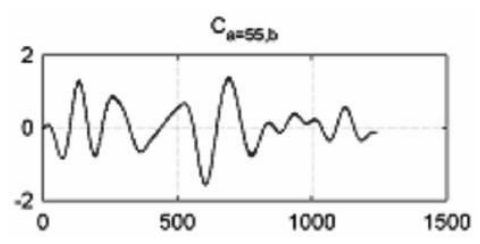

(6)

Fig. 9. Maps (fragments (1), (4)) and linear sections (fragments (2), (3), (5), (6)) of the wavelet coefficients of the distributions of the module $\left|S_{4}\right|$ (fragments (1) - (3)) and phase $\operatorname{ArgS}_{4}$ (fragments (3) - (6)) of the "two-point" 4th parameter of the Stokes vector of the microscopic image of the polycrystalline liquor film for the phase section $\varphi=1,2$.

Analysis of the obtained 3D Stokes-correlometric mapping data found:

1. The main polarimetric manifestations of the correlation matching along the directions of the optical axes $\left(\left|S_{4}\right|\right)$ and birefringence $\left(\mathrm{ArgS}_{4}\right)$ of the polycrystalline network of the optically anisotropic liquor layer is the formation of the distributions of the magnitude of MDCS $\left|S_{4}\right|(\varphi, x, y)$ and PDCS $\operatorname{Arg}_{4}(\varphi, x, y) \quad$ (fig. 1, fig. 2, fig. 4, fig. 5, fig. 7, fig. $8)$.

2. For each of the coordinate distributions $\left|S_{4}\right|(\varphi, x, y)$ and $\operatorname{Arg}_{4}(\varphi, x, y)$ within the partial phase cross sections $\varphi_{i}=0,4$ pad; 0,8pad; 1,2 pad, an individual statistical inherent (Fig. 1, Fig. 4, Fig. 7) and topographic structure - in fig. 2 , fig. 5 , fig. 8 .

3. With increasing ( $\uparrow$ ) of the phase cross section ( $\varphi \uparrow$ ) of the field of complex amplitudes, the ranges of changes in the local values of the module $\left|S_{4}\right|(\varphi, x, y)$ and phase $\operatorname{Arg}_{4}(\varphi, x, y)$ increase - fig. 2, fig. 5, fig. 8 respectively.

In the process of crystallization of the liquor film and its partial layers, various crystalline fractions are formed. Among them, two main ones can be distinguished that prevail over other biochemical compounds in concentration. The first one is large-scale $(20 \mu m \div 100 \mu m)$ needle-shaped with spatially oriented directions of the optical axes linearly birefringent albumin crystals $(\sim 70 \%-80 \%)$. The second is small-scale $(5 \mu m \div 20 \mu m)$ spherulite globulin crystals $(\sim 20 \%-30 \%)$, which are characterized by phase modulation of circularly polarized components of the laser radiation amplitude, or circular birefringence. Thus, a predominantly polycrystalline dendritic phase-modulating $(\delta)$ network of albumin crystals with randomly located directions $(\rho)$ of optical axes is formed in the planar partial layers of the liquor film.

In the relationships between the module and the DCS phase are defined. In particular, MDCS is determined by the directions of the optical axes of the needle crystals $\left|S_{4}\right|\left(r_{1}, r_{2}\right) \leftrightarrow\left(\rho_{1}, \rho_{2}\right)$, and the PDCS value is associated with the 
phase modulation of the orthogonal components of the amplitude by optically anisotropic dendritic and spherulite crystals $\operatorname{ArgS}_{4}\left(r_{1}, r_{2}\right) \leftrightarrow\left(\delta_{1}, \delta_{2}\right)$. As a result, in each such plane of the polarization-inhomogeneous object field of the polycrystalline liquor film, an individual coordinate $(x, y)$ distribution of the magnitude of the module $\left|S_{4}\right|(x, y)$ and phase $\operatorname{ArgS}_{4}(x, y)$ is formed.

Using the reference wave by applying the digital holographic reconstruction algorithm, the layer-by-layer distributions $\left|S_{4}\right|(\varphi, x, y)$ and $\operatorname{ArgS}_{4}(\varphi, x, y)$ the 4th parameter of the Stokes vector of the object field of the polycrystalline network of the liquor film are determined.

For small values of the phase cross section $\varphi=0,4 \mathrm{pad}$, the most probable events are the interaction of laser radiation with individual needle and spherulite crystals. From the point of view of the optical-geometric structure of such a network in a given plane:

- a certain spatial-determined distribution of orientations of the optical axes with a small dispersion of the scatter of their values ( $\Delta \rho / \rho \prec \prec 1$ ) is realized for several directions of crystalline growth. In other words, for neighboring points ( $r_{1}$ and $\left.r_{2}\right)$ in a given plane, the condition is most likely $\rho\left(r_{1}\right)-\rho\left(r_{2}\right) \rightarrow 0$. As a result, the set of local Stokes correlometric domains with extreme values $\left|S_{4}\right|(\varphi, \Delta x, \Delta y) \rightarrow 1$ prevails in the MDCS $\left|S_{4}\right|(\varphi, x, y)$ distribution map, fig. 1 (fragments (1), (2)), fig. 2 (fragment (1)).

- a coordinate distribution of phase shifts $(\delta(x, y))$ is formed between linearly (needle-shaped crystals) and circularly (spherulite crystals) orthogonally polarized components of the laser radiation amplitude with an insignificant modulation amplitude $\Delta \delta \prec 0,4 p a d$. As a result, the $\operatorname{DCS} \operatorname{Arg}_{4}(\varphi, x, y)$ phase distribution map is predominantly correlation-consistent sections with extreme values $\operatorname{ArgS}_{4}(\varphi, \Delta x, \Delta y) \rightarrow 1$, - fig. 1 (fragments (3), (4)), fig. 2 (fragment (2)).

- due to the presence of a certain scatter in the orientational $\bar{\rho} \pm \Delta \rho$ and phase $\bar{\delta} \pm \Delta \delta$ components of the polycrystalline network of the liquor film, $\left|S_{4}\right|(\varphi, x, y)$ and $\operatorname{ArgS}_{4}(\varphi, x, y)$ distributions contain ensembles of Stokescorrelometric domains with other and extreme values of $0 \prec\left|S_{4}\right|(\varphi, \Delta x, \Delta y) \prec 1$ and $0 \prec \operatorname{Arg}_{4}(\varphi, \Delta x, \Delta y) \prec 1$.

Within the framework of the statistical approach to the analysis of the distributions of MDCS cards (Fig. 1, fragments (1), (2), fig. 2, fragment (1)) and PDCS (fig. 1, fragments (3), (4), fig. 2, fragment (2)), one should expect a significant advantage of the values of statistical moments of higher orders (asymmetry and excess) $Z_{3 ; 4}\left(\left|S_{4}\right|(\varphi=0,4\right.$ pad, $\left.\mathrm{x}, \mathrm{y})\right), Z_{3 ; 4}\left(\operatorname{Arg} S_{4}(\varphi=0,4\right.$ pad, $\left.\left.\mathrm{x}, \mathrm{y})\right)\right)$ over the average $\left(Z_{1}\right)$ and dispersion $\left(Z_{2}\right)$.

The results of digital holographic reproduction of polarization-correlation maps $\left|S_{4}\right|(\varphi, x, y)$ and $\operatorname{Arg} S_{4}(\varphi, x, y)$ for large values of phase cross sections ( $\varphi=0,8 \mathrm{pad} ; 1,2 \mathrm{pad})$ of three-dimensional distribution of the field of complex amplitudes of laser radiation correspond to the physical situation, where the probability of interaction of partial waves with several optically anisotropic crystalline formations of the polycrystalline network of the liquor increases. As a result, the influence on the polarization-correlation structure of such an object field increases in the range of variation in the directions of the optical axes $\Delta \rho(\varphi, x, y) \uparrow$ of birefringent crystals, as well as the depth of phase modulation $\Delta \delta(\varphi, x, y) \uparrow$. Such a transformation of the optical-geometric structure of the polycrystalline component in these layers of the liquor film manifests itself in an increase in the coordinate decorrelation between the values $\rho(\varphi, x, y)$ and $\delta(\varphi, x, y)$, which is optically manifested in the redistribution of the extreme values of the module $\left|S_{4}\right|(\varphi, x, y)=1$ and phase $\operatorname{Arg}_{4}(\varphi, x, y)=1,75 \mathrm{pad}$ to a wider range of their variation $0 \prec\left|S_{4}\right|(\varphi, x, y) \prec 1$ and $0 \prec \operatorname{Arg} S_{4}(\varphi, x, y) \prec 1,75 \mathrm{pad}$ - fig. 4, fig. 7. In addition, in these phase sections, the quantity, area, size and range of changes of the module $0 \prec\left|S_{4}\right|(\varphi, \Delta x, \Delta y) \prec 1$ and phase $0 \prec \operatorname{Arg}_{4}(\varphi, x, y) \prec 1,75$ pad within the Stokes-correlometric domains grows - fig. 5 and fig. eight. 
Quantitatively (within the limits of the statistical analysis of the maps of the module and the DCS phase) this is manifested in opposite trends in the magnitude of the statistical moments of the 1st - 4th orders characterizing the distribution of the magnitude of the module $\left|S_{4}\right|(\varphi, \mathrm{x}, \mathrm{y})$ and phase $\operatorname{Arg}_{4}(\varphi, \mathrm{x}, \mathrm{y})$ - the average and dispersion grow $\left\{Z_{1 ; 2}\left(\left|S_{4}\right|(\varphi, \mathrm{x}, \mathrm{y})\right) ; Z_{1 ; 2}\left(\operatorname{ArgS}_{4}(\varphi, \mathrm{x}, \mathrm{y})\right)\right\} \uparrow ;$ statistical moments of higher orders, on the contrary, decrease $\left\{Z_{3 ; 4}\left(\left|S_{4}\right|(\varphi, \mathrm{x}, \mathrm{y})\right) ; Z_{3 ; 4}\left(\operatorname{Arg}_{4}(\varphi, \mathrm{x}, \mathrm{y})\right)\right\} \downarrow$ - table 1 .

Table 1. Statistical moments $Z_{i=1 ; 2 ; 3 ; 4}$ that characterize the distribution of magnitude $\left|S_{4}\right|$ and $\operatorname{Arg} S_{4}$ microscopic image of a polycrystalline film of liquor

\begin{tabular}{|c|c|c|c|c|c|}
\hline \multicolumn{3}{|c|}{$\left|S_{4}\right|$} & \multicolumn{3}{|c|}{$\mathrm{ArgS}_{4}$} \\
\hline \multirow[t]{4}{*}{$\varphi=0,4$} & $Z_{1}$ & 0,54 & \multirow[t]{4}{*}{$\varphi=0,4$} & $Z_{1}$ & 0,93 \\
\hline & $Z_{2}$ & 0,09 , & & $Z_{2}$ & 0,07 \\
\hline & $Z_{3}$ & 0,53 & & $Z_{3}$ & 0,81 \\
\hline & $Z_{4}$ & 1,47 & & $Z_{4}$ & 1,79 \\
\hline \multirow[t]{4}{*}{$\varphi=0,8$} & $Z_{1}$ & 0,39 & \multirow[t]{4}{*}{$\varphi=0,8$} & $Z_{1}$ & 0,84 \\
\hline & $Z_{2}$ & 0,12 & & $Z_{2}$ & 0,11 \\
\hline & $Z_{3}$ & 0,44 & & $Z_{3}$ & 0,73 \\
\hline & $Z_{4}$ & 1,16 & & $Z_{4}$ & 1,41 \\
\hline \multirow[t]{4}{*}{$\varphi=1,2$} & $Z_{1}$ & 0,21 & \multirow[t]{4}{*}{$\varphi=1,2$} & $Z_{1}$ & 0,51 \\
\hline & $Z_{2}$ & 0,15 & & $Z_{2}$ & 0,13 \\
\hline & $Z_{3}$ & 0,38 & & $Z_{3}$ & 0,61 \\
\hline & $Z_{4}$ & 0,89 & & $Z_{4}$ & 1,24 \\
\hline
\end{tabular}

A comparative analysis of the change in the magnitude of the statistical moments of the 1st - 4th orders of magnitude of the 3D distribution of the magnitude $\left|S_{4}\right|$ and $A r g S_{4}$ the "two-point" 4th parameter of the Stokes vector of the microscopic image of the polycrystalline liquor film in different phase sections of the liquor film revealed significant differences between them - $\left\{\begin{array}{l}\left|S_{4}\right| \Rightarrow \Delta Z_{1}(\varphi)=2,7 ; \Delta Z_{2}(\varphi)=2,3 ; \Delta Z_{3}(\varphi)=1,9 ; \Delta Z_{4}(\varphi)=1,7 ; \\ A r g S_{4} \Rightarrow \Delta Z_{1}(\varphi)=1,8 ; \Delta Z_{2}(\varphi)=1,9 ; \Delta Z_{3}(\varphi)=1,3 ; \Delta Z_{4}(\varphi)=1,4 ;\end{array}\right.$

As can be seen, the statistical moments of the 1st and 2nd orders characterizing the distribution of the magnitude of the module $\left|S_{4}\right|(\varphi, \mathrm{x}, \mathrm{y}) \quad\left(Z_{1 ; 2}\right.$ - differences for different phase cross sections are $2.3-2.7$ times $)$ and phases $\operatorname{Arg} S_{4}(\varphi, \mathrm{x}, \mathrm{y})$ $\left(Z_{1 ; 2}\right.$ - differences for different phase cross sections are 1.8 - 1.9 times) of the polarization-inhomogeneous object field of laser radiation.

The results of a scale-selective wavelet analysis ${ }^{12-24}$ of the coordinate distributions of the magnitude of the module $\left|S_{4}\right|(\varphi, \mathrm{x}, \mathrm{y})$ and phase $\operatorname{ArgS}_{4}(\varphi, \mathrm{x}, \mathrm{y})$ of the 4th parameter of the "two-point" Stokes vector in various phase ( $\left.\varphi\right)$ planes of the polarization-inhomogeneous object field of a polycrystalline liquor film are represented by a series of twodimensional (fragments (1), (4)) and linear (fragments (2), (3), (5), (6)) dependences of the amplitudes of the wavelet coefficients in fig. 3 ( $\varphi=0,4$ pad ), fig. $6(\varphi=0,8$ pad $)$ and fig. $9(\varphi=1,2 p a \partial)$.

Analysis of the data revealed: 
1. The individual structure of two-dimensional maps of wavelet coefficients (fragments (1), (4)) characterizing different-scale linear dependences of the amplitude of the wavelet coefficients $C_{a=15, b}$ and $C_{a=55, b}$ obtained for the scale $\mathrm{a}=15$ and $\mathrm{a}=55$ MHAT functions - fragments (2), (3), (5), (6).

2. The amplitude of the linear dependences $C_{a=55, b}$ of the wavelet coefficients characterizing the large-scale structure of the distributions of the module $\left|S_{4}\right|(\varphi, x, y)$ and phase $\operatorname{ArgS}_{4}(\varphi, x, y)$ in the phase section $\varphi=0,4$ pad is larger (23 times) than the fluctuations of the same parameter $C_{a=15, b}$ that characterizes the MDCS and PDCS maps fragments (2), (3) and (5), (6).

3. For large phase cross sections ( $\varphi=0,8$ pad; 1,2pad ) of the polarization-inhomogeneous field of the network of dendritic crystals of the liquor film, the range of variation in the amplitude of the linear dependences of the wavelet coefficients $C_{a=15, b} \downarrow$ of the small-scale structure of the coordinate distributions $\left|S_{4}\right|(\varphi, x, y)$ and $\operatorname{Arg} S_{4}(\varphi, x, y)$ decreases (fig. 3, fig. 6, fig. 9, fragments (2), (5)); for large-scale coefficients $C_{a=55, b} \uparrow$ is growing, - Fig. 3, fig. 6, fig. 9, fragments (3), (6).

The established regularities can be associated with the fact that the wavelet coefficient value $C_{a, b}$ is determined by the degree of correlation consistency of the dimensions of the Stokes-correlometric domain $\left(\left|S_{4}\right|(\varphi, \Delta x, \Delta y)\right.$ or $\left.\operatorname{ArgS}_{4}(\varphi, \Delta x, \Delta y)\right)$ with the size of the MHAT function window, which scans the coordinate distributions of $\left|S_{4}\right|(\varphi, x, y)$ and $\operatorname{ArgS}_{4}(\varphi, x, y)$ in rows. The smallest sizes of the large-scale components of the MDCS and PDCS cards are formed in phase sections of the field of complex amplitudes $E_{x}(\varphi, x, y), E_{x}(\varphi, x, y)$ polycrystalline liquor film with an insignificant level $\varphi=0,4$ pad (fig. 2). Therefore, an insignificant level of correlation between the dimensions of the window ( $a=55$ ) of the MHAT function and the dimensions of the domains of Stokes correlometric maps is realized in this plane. As a result, the amplitude of the change in the linear dependence $C_{a=55, b}$ is characterized by the minimum range of change - $-1 \prec C_{a=55, b} \prec 1$ (fig. 3, fragments (2), (5)).

The increase $\varphi \uparrow$ allows us to estimate the correlation consistency of the parameters of optical-anisotropic formations on large geometric scales. In accordance with this, the level of correlation between the window parameters ( $a=55$ ) of the MHAT function and the sizes $\left|S_{4}\right|(\varphi, \Delta x, \Delta y)$ or $\operatorname{ArgS}_{4}(\varphi, \Delta x, \Delta y)$ increases. As a result, the range of changes in the amplitude of wavelet coefficients ( $-2 \prec C_{a=55, b} \prec 2$, fig. 6, fragments (2), (5) and $-3 \prec C_{a=55, b} \prec 3$, fig. 9, fragments (2), (5)), which characterize the coordinate distributions of the magnitude of the module, is significantly increased (Fig. 5 , Fig. 8, fragments (1)) and phases (fig. 5, fig. 8, fragments (2)) of DCS in phase sections $\varphi=0,8$ pad and $\varphi=1,2$ pad. At smaller geometric scales of the MHAT, the amlitude dynamics of the linear dependences $C_{a=15, b}$ of the distributions of the wavelet coefficients characterizing the maps $\left|S_{4}\right|(\varphi, x, y)$ and $\operatorname{Arg} S_{4}(\varphi, x, y)$ (fig. 2, fig. 5, fig. 8) for the different phase planes of the field of complex amplitudes $E_{x}(\varphi, x, y)$ and $E_{x}(\varphi, x, y)$ polycrystalline liquor film is much smaller, - fig. 3 ( $-0,5 \prec C_{a=55, b} \prec 0,5$ ), fig. 6 ( $\left.-1 \prec C_{a=55, b} \prec 1\right)$, fig. 9 ( $-1,5 \prec C_{a=55, b} \prec 1,5$ ), fragments (3), (6).

This fact can be related to the fact that the concentration, and, accordingly, the number of small-scale optically anisotropic spherulitic formations, is significantly lower compared to the number of linearly birefringent dendritic crystals. Due to this, the number of Stokes-correlometric domains and their geometric dimensions vary slightly in different phase planes of the object field of laser radiation (fig. 2, fig. 5, fig. 8, fragments (2)). As a result, the level of correlation consistency between the window size $(a=15)$ of the MHAT function and the area $\left|S_{4}\right|(\varphi, \Delta x, \Delta y)$ or $\operatorname{ArgS}_{4}(\varphi, \Delta x, \Delta y)$ is much smaller compared to the large scale of the wavelet function. 
The values of the statistical $Z_{i=1 ; 2 ; 3 ; 4}$ moments of the 1 st - 4th orders characterize the linear dependences $C_{a=15, b}$ and $C_{a=55, b}$ amplitudes of the wavelet coefficients of the distribution of the magnitude $\left|S_{4}\right|$ and $A r g S_{4}$ in different phase sections are shown in table 2.

Table 2. Statistical moments $Z_{i=1 ; 2 ; 3 ; 4}$ that characterize the linear dependences $C_{a=15, b}$ and $C_{a=55, b}$ amplitudes of the wavelet coefficients of the distribution of magnitude $\left|S_{4}\right|$ and $\operatorname{ArgS}_{4}$

\begin{tabular}{|c|c|c|c|c|c|c|c|}
\hline \multicolumn{4}{|c|}{$\left|S_{4}\right|$} & \multicolumn{4}{|c|}{$\mathrm{ArgS}_{4}$} \\
\hline$\varphi_{i}$ & $Z_{i}$ & $C_{a=15, b}$ & $C_{a=55, b}$ & $\varphi_{i}$ & $Z_{i}$ & $C_{a=15, b}$ & $C_{a=55, b}$ \\
\hline \multirow[t]{4}{*}{$\varphi=0,4$} & $Z_{1}$ & 0,06 & 0,11 & \multirow[t]{4}{*}{$\varphi=0,4$} & $Z_{1}$ & 0,08 & 0,15 \\
\hline & $Z_{2}$ & 0,21 & 0,63 & & $Z_{2}$ & 0,29 & 0,77 \\
\hline & $Z_{3}$ & 0,51 & 0,71 & & $Z_{3}$ & 0,42 & 0,86 \\
\hline & $Z_{4}$ & 0,42 & 0,65 & & $Z_{4}$ & 0,32 & 0,73 \\
\hline \multirow[t]{4}{*}{$\varphi=0,8$} & $Z_{1}$ & 0,09 & 0,21 & \multirow[t]{4}{*}{$\varphi=0,8$} & $Z_{1}$ & 0,11 & 0,26 \\
\hline & $Z_{2}$ & 0,34 & 1,15 & & $Z_{2}$ & 0,41 & 1,31 \\
\hline & $Z_{3}$ & 0,39 & 0,52 & & $Z_{3}$ & 0,31 & 0,64 \\
\hline & $Z_{4}$ & 0,33 & 0,41 & & $Z_{4}$ & 0,24 & 0,58 \\
\hline \multirow{4}{*}{$\begin{array}{l}\varphi=1,2 \\
\varphi=0,4\end{array}$} & $Z_{1}$ & 0,12 & 0,37 & \multirow{4}{*}{$\begin{array}{l}\varphi=1,2 \\
\varphi=0,4\end{array}$} & $Z_{1}$ & 0,14 & 0,42 \\
\hline & $Z_{1}$ & 0,42 & 2,12 & & $Z_{1}$ & 0,52 & 2,34 \\
\hline & $Z_{2}$ & 0,18 & 0,35 & & $Z_{2}$ & 0,14 & 0,47 \\
\hline & $Z_{3}$ & 0,24 & 0,33 & & $Z_{3}$ & 0,16 & 0,41 \\
\hline
\end{tabular}

A comparative analysis of the changes in the statistical moments of the first and fourth orders characterizing the linear dependences of the amplitude of the wavelet coefficients at different scales of the MHAT function in different phase sections of the 3D distributions of the MDCS and PDCS object field of the polycrystalline liquor film revealed significant differences between them:

- $\left|S_{4}\right|=\left\{\begin{array}{l}a=15 \Rightarrow \Delta Z_{1}(\varphi)=2 ; \Delta Z_{2}(\varphi)=2 ; \Delta Z_{3}(\varphi)=2,8 ; \Delta Z_{4}(\varphi)=2,7 ; \\ a=55 \Rightarrow \Delta Z_{1}(\varphi)=3,4 ; \Delta Z_{2}(\varphi)=3,4 ; \Delta Z_{3}(\varphi)=2,2 ; \Delta Z_{4}(\varphi)=2 ;\end{array}\right.$

- $\quad \operatorname{ArgS}_{4}=\left\{\begin{array}{l}a=15 \Rightarrow \Delta Z_{1}(\varphi)=1,8 ; \Delta Z_{2}(\varphi)=1,8 ; \Delta Z_{3}(\varphi)=2 ; \Delta Z_{4}(\varphi)=2 ; \\ a=55 \Rightarrow \Delta Z_{1}(\varphi)=2,8 ; \Delta Z_{2}(\varphi)=3,1 ; \Delta Z_{3}(\varphi)=1,8 ; \Delta Z_{4}(\varphi)=1,8 .\end{array}\right.$

As can be seen, the most sensitive to changes in the orientation-phase structure at different scales of the geometric dimensions of the polycrystalline network of the liquor film are the statistical moments of the first and second orders, characterizing the distribution of the amplitude of the linear sections of the wavelet coefficients of the module ( $Z_{1 ; 2}\left(\varphi,\left|S_{4}\right|\right)$ - the differences for different phase sections are - 3.4 times $)$ and phases $\left(Z_{1 ; 2}\left(\varphi, \operatorname{Arg} S_{4}\right)\right.$ - differences for different phase cross sections are - 2.8 - 3.1 times) of the 4th parameter of the Stokes vector of the polarizationinhomogeneous object field of laser radiation for scale MHAT function $a=55$.

Thus, we can state that the use of additional scale-selective wavelet analysis (fig. 3, fig. 6, fig. 9) of the coordinate distributions of MDCS and PDCS (fig. 1, fig. 2, fig. 4, fig. 5, fig. 7, fig. 8) in the phase $(\varphi=0,4 p a \partial, \varphi=0,8 p a \partial$ and $\varphi=1,2$ pad) sections of the microscopic image of the volume of the polycrystalline liquor film increases the sensitivity of the $3 \mathrm{D}$ polarization-correlation mapping method to $25 \%-30 \%$ (table 1 and table 2 ). 


\section{CONCLUSIONS}

The 3D Stokes-correlometry method of polarization-inhomogeneous object fields of biological fluid films based on a reference laser wave and layer-by-layer digital holographic reproduction of complex amplitude distributions with subsequent determination of the corresponding module maps and phase parameters of the "two-point" Stokes vector was experimentally tested.

The relationships between the trends in the magnitude of statistical moments of the 1st -4 th orders are determined, which characterize the layer-by-layer distributions of 3D maps of the module and the phase of the parameters of the "two-point" Stokes vector of laser radiation transformed by networks of biochemical crystals of liquor films.

Additional possibilities of expanding the functionality and sensitivity of the 3D polarization-correlation mapping method for polycrystalline networks using wavelet analysis of the coordinate distributions of the magnitude and phase parameter of the "two-point" Stokes vector of the object fields of films of biological fluids of human organs have been investigated.

In the framework of the comparative studies of linear dependences of the amplitude of the wavelet coefficients of the layer-by-layer coordinate distributions of the magnitude of the modulus and phase of the parameters of the "two-point" Stokes vector of the object field of the dendritic and spherulite network of polycrystalline cerebrospinal fluid films, the values and ranges of statistical parameters that characterize it are established.

\section{REFERENCES}

[1]. Wang X. Propagation of polarized light in birefringent turbid media: a Monte Carlo study / X. Wang, L.-H. Wang // J. Biomed. Opt. - 2002. - Vol. 7. - P. 279-290.

[2]. Tuchin V. V. Handbook of optical biomedical diagnostics / V. V. Tuchin. - Bellingham : SPIE Press, 2002. - 1110 p.

[3]. Yao G. Two-dimensional depth-resolved Mueller matrix characterization of biological tissue by optical coherence tomography / G. Yao, L. V. Wang // Opt. Lett. - 1999. - V. 24. - P. 537-539.

[4]. Tower T. T. Alignment Maps of Tissues: I. Microscopic Elliptical Polarimetry / T. T. Tower, R. T. Tranquillo // Biophys. J. - 2001. - Vol. 81. - P. 2954-2963.

[5]. Lu S. Interpretation of Mueller matrices based on polar decomposition / S. Lu, R. A. Chipman // J. Opt. Soc. Am. A. 1996. - Vol. 13. - P.1106-1113.

[6]. Ghosh Nirmalya. Techniques for fast and sensitive measurements of two-dimensional birefringence distributions / Nirmalya Ghosh, I. Alex Vitkin // Journal of Biomedical Optics. - 2011. - № 16(11). - P. 110801.

[7]. V. V. Tuchin, L. Wang, and D. A . Zimnyakov, Optical Polarization in Biomedical Applications, New York, USA (2006).

[8]. Angelsky, O.V., Hanson, S.G., Maksimyak, P.P., Maksimyak, A.P., Zenkova, C.Yu., Polyanskii, P.V., Ivanskyi, D.I., "Influence of evanescent wave on birefringent microplates," (2017) Optics Express, 25 (3), pp. 2299-2311.

[9]. 2. Angelsky, O.V., Ushenko, Y.A., Dubolazov, A.V., Telenha, O.Yu., "The interconnection between the coordinate distribution of mueller-matrixes images characteristic values of biological liquid crystals net and the pathological changes of human tissues,"(2010) Advances in Optical Technologies, art. no. 130659.

[10]. Bekshaev, A.Ya., Angelsky, O.V., Sviridova, S.V., Zenkova, C.Yu., "Mechanical action of inhomogeneously polarized optical fields and detection of the internal energy flows," (2011) Advances in Optical Technologies, art. no. 723901.

[11]. Angelsky, O.V., Maksimyak, P.P., Perun, T.O., "Optical correlation method for measuring spatial complexity in optical fields," (1993) Optics Letters, 18 (2), pp. 90-92.

[12]. Angelsky, O.V., Ushenko, A.G., Ushenko, Y.A., Pishak, V.P., "Statistical and fractal structure of biological tissue mueller matrix images," (2007) Optical Correlation Techniques and Applications, pp. 213-265.

[13]. Angelsky, O.V., Ushenko, A.G., Pishak, V.P., Burkovets, D.N., Yermolenko, S.B., Pishak, O.V., Ushenko, Yu.A., "Coherent introscopy of phase-inhomogeneous surfaces and layers," (2000) Proceedings of SPIE - The International Society for Optical Engineering, 4016, pp. 413-418.

[14]. Angelsky, O.V., "Optical correlation techniques and applications,"(2007) Optical Correlation Techniques and Applications, pp. 1-270.

[15]. Angelsky, O.V., Maksimyak, P.P., "Optical diagnostics of slightly rough surfaces,” (1992) Applied Optics, 31 (1), pp. 140-143.

[16]. Angelsky, O.V., Maksimyak, P.P., "Polarization-interference measurement of phase-inhomogeneous objects," (1992) Applied Optics, 31 (22), pp. 4417-4419.

[17]. Angelsky, O. V., Bekshaev, A. Ya., Maksimyak, P. P., Maksimyak, A. P., Hanson, Steen Grüner, "Low-temperature laser-stimulated controllable generation of micro-bubbles in a water suspension of absorptive colloid particles," Optics Express (2018), Vol. 26, No. 11. pp. 13995-14009. 
[18]. Ushenko, Yu.A., Tomka, Yu.Ya., Dubolazov, A.V., Telen'ga, O.Yu. Diagnostics of optical anisotropy changes in biological tissues using Müller matrix (2011) Quantum Electronics, 41 (3), pp. 273-277.

[19]. Ushenko, Yu.A., Tomka, Yu.Ya., Dubolazov, A.V. Laser diagnostics of anisotropy in birefringent networks of biological tissues in different physiological conditions (2011) Quantum Electronics, 41 (2), pp. 170-175.

[20]. Ushenko, Y.A., Dubolazov, O.V., Karachevtsev, A.O. Statistical structure of skin derma Mueller matrix images in the process of cancer changes (2011) Optical Memory and Neural Networks (Information Optics), 20 (2), pp. 145-154.

[21]. Ushenko, V.A., Dubolazov, A.V. Correlation and self similarity structure of polycrystalline network biological layers mueller matrices images (2013) Proceedings of SPIE - The International Society for Optical Engineering, 8856, 88562D.

[22]. Ushenko, A.G., Dubolazov, A.V., Ushenko, V.A., Novakovskaya, O.Y. Statistical analysis of polarizationinhomogeneous Fourier spectra of laser radiation scattered by human skin in the tasks of differentiation of benign and malignant formations (2016) Journal of Biomedical Optics, 21 (7), 071110.

[23]. Ushenko, Y.A., Dubolazov, A.V., Angelsky, A.P., Sidor, M.I., Bodnar, G.B., Koval, G., Zabolotna, N.I., Smolarz, A., Junisbekov, M.S. Laser polarization fluorescence of the networks of optically anisotropic biological crystals (2013) Proceedings of SPIE - The International Society for Optical Engineering, 8698, 869809.

[24]. Ushenko, Yu.A., Bachynsky, V.T., Vanchulyak, O.Ya., Dubolazov, A.V., Garazdyuk, M.S., Ushenko, V.A. Jonesmatrix mapping of complex degree of mutual anisotropy of birefringent protein networks during the differentiation of myocardium necrotic changes (2016) Applied Optics, 55 (12), pp. B113-B119. 